\title{
Direct Blind Deconvolution of FIR Multiple-Input Multiple-Output Systems
}

\author{
Yujiro Inouye $^{1}$ and Ruey-wen Liu ${ }^{2}$
}

\author{
${ }^{1}$ Department of Electronic and Control Systems Engineering, Shimane University, 1060 Nishikawatsu, \\ Matsue, Shimane 690-8504, Japan. E-mail: inouye@riko.shimane-u.ac.jp \\ Department of Electrical Engineering, University of Notre Dame, Notre Dame, IN 46556, USA. \\ E-mail: liu.1@nd.edu
}

\begin{abstract}
We consider the blind deconvolution problem of FIR multiple-input multiple-output channel systems with or without transmission zeros at the origin. The number of the outputs (of a system) is assumed to be greater than the number of the inputs. A channel system is said to be equalizable if there exists an FIR equalizer which equalizes it. It is shown that any equalizable FIR system can be expressed as a product of an irreducible system and a paraunitary system. Based on this fact, it is shown that any equalizable FIR system can be converted to a paraunitary system through the whitening of the observations using the second-order statistics. When the original FIR system has no transmission zero at the origin, the paraunitary system obtained through the whitening of the observations becomes a constant (static) system.
\end{abstract}

\section{INTRODUCTION}

Blind identification and deconvolution of finite impulse response (FIR) systems based on second-order statistics (SOS) have recently received attention in various fields such as digital communications, image processing and neural information processing [1]. Many recently published papers, however, concentrate on blind identification of single-input multiple-output (SIM0) FIR systems [2]-[6]. Some recent papers consider blind identification of multiple-input multipleoutput (MIMO) FIR systems [7]. All of these papers are essentially based on a two-step procedure: First, identify a channel system and then design an equalizer. A fundamental restriction is that the channel system treated in these papers does not allow transmission zeros at the origin $z=0$ in the $z$-plane (where $z$ is a complex variable standing for the delay operation in the time domain). This means that all source signals directly transmitted by different multiple users (through a propagation medium) are received simultaneously by an antenna array. This requirement is very restrictive in mobile communications. Some source signals may reach the antenna array with delays. Moreover, identification of channel characteristics is not our final goal, but an intermediate step for the retrieval of source signals.

In this paper, we consider a class of FIR MIMO channel systems called equalizable channels. This class of equalizable channels allows transmission zeros at the origin $z=0$, and hence it does not require the arrival time of sources to an antenna array to be the same. The number of inputs $n$ is less than or equal to the number of outputs $\mathrm{m}$. We formulate the blind equalization problem of an FIR channel system, and show a necessary and sufficient condition for the existence of an FIR equalizer. To analyze FIR channel systems, we show that any equalizable FIR system can be written as a product of an irreducible FIR system and a paraunitary FIR system. Based on this fact, we show that any equalizable FIR channel system can be reduced to a paraunitary FIR channel system by a whitener of the channel system.

If the original FIR channel system has no transmission zero at the origin, then the above paraunitary FIR channel system is a static system. This result was first by Slock [8] for the single input case, and extended to the multiple input case by Gorokhov and Loubaton [7]. However, this result was obtained under the two assumptions that the original channel system is irreducible and column-reduced [7]. It is important to emphasize that this result will be shown without the assumption on the channel system to be irreducible or column-reduced in this contribution. This is possible because these conditions are important to blind channel identification, but not to direct channel equalization. 


\section{ASSUMPTIONS AND PRELIMINARIES}

We consider an FIR MIMO channel-filter system described as shown in Fig. 1:

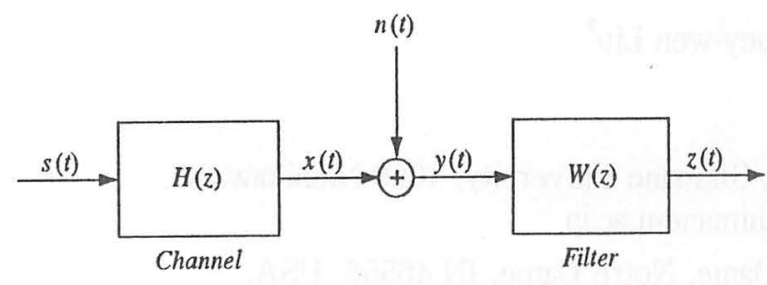

Fig. 1: A Channel-Filter System

where

$\begin{array}{ll}s(t) & n \text {-vector of source signals } \\ y(t) & m \text {-vector of channel output signals } \\ n(t) & m \text {-vector of noises } \\ z(t) & q \text {-vector of filter outputs } \\ H(z) & \text { FIR channel system, } H(z) \in[z]^{m \times n} \\ W(z) & \text { FIR filter system, } W(z) \in C[z]^{q \times m} .\end{array}$

Here $C[z]^{m \times n}$ denotes the set of all $m \times n$ matrices with components that are polynomials in $z$ with complex coefficients. The channel system $H(z)$ is represented by its finite impulse response (FIR) $\left\{H_{k} \in C^{m \times n} \mid k=\overline{0, K}\right\}$. Its input-output relation is given by

$$
y(t)=\sum_{k=0}^{K} H_{k} s(t-k)+n(t), \quad t \in Z .
$$

We may write (1) as

$$
y(t)=H(z) s(t)+n(t), \quad t \in Z,
$$

where $H(z) \in C[z]^{m \times n}$ is the transfer function of the channel system, which is the $z$-transform of its impulse response $\left\{H_{\mathrm{k}}\right\}$, i.e.,

$$
H(z)=\sum_{k=0}^{K} H_{k} z^{k}, \quad z \in C .
$$

If $H_{K} \neq 0$, then the degree of the channel matrix is $\operatorname{deg} H(z)=K$, which is also the order of the channel system. Setting $z=e^{-j \omega}$ in (3), it is obtained the Fourier transform $H\left(e^{-j \omega}\right)$ of $\left\{H_{k}\right\}$.
To process the output signals, we cascade the channel output with another FIR system, called a filter $W(z)$. Similarly, $W(z)$ is represented by an FIR sequence $\left\{W_{k} \in C^{q \times m} \mid k=\overline{0, L}\right\}$, and its $z$-transform is denoted by $W(z)$. Its input-output relation is described by

$$
z(t)=\sum_{k=0}^{L} W_{k} y(t-k), \quad t \in Z
$$

or, $z(t)=W(z) y(t)$. In this paper, we consider the two cases of filters: $q=n$, and $q=m-n$ when $m>n$.

The total system input $s(t)$ and output $z(t)$ are related by

$$
z(t)=G(z) s(t)+W(z) n(t),
$$

where the total system transfer function is given by

$$
G(z):=W(z) H(z)
$$

The objective of blind equalization is to design a filter $W(z)$ that recovers the original source signals $s(t)$ only from the observations of the channel-output $y(t)$. Since we cannot observe the source signals but only the channel-output, there are inherent ambiguities in the solution to this problem. Taking these ambiguities into account, we introduce the following notions for linear time-invariant (LTD) systems.

Definition 1: A scalar function $g(z)$ of a complex variable $z$ is said to be monomial if it can be represented as $g(z)=d z$, for some $d \in C$ and some non-negative integer $\ell$. An LTI system with $n$ inputs and $n$ outputs is said to be transparent if its transfer function matrix $G(z)$ has a single nonzero monomial entry in each row and each column.

It should be noted that an LTI system is transparent if and only if its transfer function $G(z)$ has a decomposition of the form

$$
G(z)=P D \Lambda(z),
$$

where $P$ is a permutation matrix, $D$ a regular diagonal matrix, and $\Lambda(z) \in \Lambda[z]^{n \times n}$. Here $\Lambda[z]^{n \times n}$ denotes the set of $n \times n$ regular diagonal matrices with diagonal entries being monic monomials.

The blind equalization problem can be formulated 
as follows: Design a filter $W(z)$, called an equalizer, without the knowledge of $H(z)$, so that the condition

$$
W(z) H(z)=P D \Lambda(z)
$$

is satisfied, i.e., the total system $G(z)=W(z) H(z)$ is transparent.

The existence of such equalizers is not guaranteed for each channel system. A channel system $H(z)$ is said to be equalizable if there exists an equalizer $W(z)$ so that the total system $G(z)$ is transparent, i.e., (8) is satisfied. A characterization of equalizable channel systems is given by Massey and Sain [12], with a slight modification. An alternative proof of Theorem 1 is given in [11].

Theorem 1: (Equalizability Condition): A necessary and sufficient condition for a channel system $H(z) \in C[z]^{m \times n}$ to be equalizable is that the greatest common divisor $(\mathrm{GCD})$ of all the $n \times n$ minors of $H(z)$ is nonzero monomial. This condition is equivalent to

$$
\operatorname{rank} H(\lambda)=n \text { for all nonzero } \lambda \in C \text {. }
$$

In general, the blind equalization problem mentioned above can be solved by higher-order statistics (HOS) of the observations. However, when $m>n=1$, i.e., the single source case, it was shown by Tong et al. [2] that it can be solved by use of only second-order statistics (SOS). Ever since then, there has been much interest in solving this problem using only the SOS of the observations or exploiting them at least to the maximum possible extent.

One of the objectives of this paper is to clarify the limitations of the power of SOS-based approaches towards the solution of the MIMO blind equalization problem. To this end, we next present the definition of the paraunitary systems and then introduce some notions on filters cascaded with the channel system $H(z)$.

Definition 2: A square transfer function $H_{P}(z) \in C[z]^{n \times n}$ is said to be paraunitary if it is unitary on the unit cycle, i.e.

$$
H_{P}\left(e^{-j \omega}\right) H_{P}^{H}\left(e^{-j \omega}\right)=I \text { for all } \omega,
$$

where the superscript $H$ denotes the complex conjugate transpose (Hermitian ) operation of a matrix. The FIR system itself is called to be paraunitary for simplicity.

In addition to equalizers, we propose four classes of filters $W(z)$ to be cascaded with $H(z) \in C[z]^{m \times n}$.

1) Whiteners: A filter $W(z) \in C[z]^{n \times m}$ is said to be a whitener for system $H(z)$ driven by a white sequence (see A2 below) if it satisfies

$$
W(z) H(z)=D H_{P}(z)
$$

where $D \in C^{n \times n}$ is a regular diagonal matrix and $H_{P}(z) \in C[z]^{n \times n}$ is paraunitary. This condition follows from the well-known fact that if both the input and the output are normalized white then the system is paraunitary.

2) Deconvolvers: A filter $W(z) \in C[z]^{n \times m}$ is said to be a deconvolver (or strict deconvolver) for $H(z)$ if it satisfies

$$
W(z) H(z)=C \Lambda(z),(\text { or } W(z) H(z)=C)
$$

where $C$ is a regular constant matrix and $\Lambda(z) \in \Lambda[z]^{n \times n}$. A channel system $H(z)$ is said to be deconvolvable (or strictly deconvolvable) if there exists a deconvolver (or strict deconvolver) for $H(z)$.

3) Blockers: A filter $W(z) \in C[z]^{(m-n) \times m}$ is said to be a blocker for $H(z)$ if its normal rank is $m-n$ and it satisfies

$$
W(z) H(z)=0 .
$$

4) Demixers: When $H(z)=H \in C^{m \times n}$ is a constant matrix, a constant filter $W \in C^{n \times m}$ is called a demixer for $H$ if it satisfies

$$
W H=\Lambda P,
$$

where $\Lambda$ is a regular diagonal matrix and $P$ a permutation matrix, i.e., the total system is transparent.

Some comments on the above notions are in order. 
1) Whitening techniques are used for both single- and multi-channel blind equalization problems.

2) Deconvolvers constitute an important class of filters. Deconvolvers eliminate the dynamically mixed effect of the channels, but unlike equalizers, do not separate the signals. It will be shown that under some mild conditions we can only design a deconvolver (but not an equalizer), if only secondorder statistics are allowed to be used. We need further to design a demixer for source separation.

3) Blockers constitute another important class of filters. It is important for two reasons. It follows from (5) that the blocker output becomes $z(t)=W(z) n(t)$. This means that we can utilize the blocker outputs to investigate the characteristics of the noise. The second reason is that the condition $W(z) H(z)=0$ means that the row vectors of $W(z)$ span the dual space $V^{\perp}$ of the space $V$ spanned by the column vectors of $H(z)$ [16]. This property was used by Tong et al. [2] in the single-channel case, and later it was exploited extensively for multichannel case, in the subspacebased approaches [4]-[7] and in the linear prediction approaches [8].

4) There are two ways to design a demixer. When the input sequence is white, higher-order statistics of the observations will be used. When the input sequence is colored, second-order statistics of observations can also be used.

Henceforth, we assume throughout the paper that:

A1) It is assumed that the second-order statistics of the noise can be estimated and removed. As such, we assume that $n(t)=0$.

A2) The input sequence $\{s(t)\}$ is a complex, zeromean, and normalized-white (both temporally and spatially) vector-valued stationary random process, i.e., $E\{s(t)\}=0$, and $E\left\{s(t+k) s^{H}(t)\right\}=I \delta(k)$ for $t \in Z$,

where $\delta(k)$ is the Kronecker delta.

\section{FACTORIZATION AND DECONVOLUTION}

An important factorization theorem for equalizable channel matrices is first presented. Then, when the input sequence is zero-mean and white, the limitation of whitening process is investigated. It is shown that a whitening process at best can deconvolve a channel, not equalize a channel. Higher-order statistics are needed for the latter. To begin with we present the definitions of irreducible, column-reduced, and unimodular systems.

Definition 3: Let $H(z) \in C[z]^{m \times n}$ be an channel system with $m \geq n$. Then,

1) $H(z)$ is said to be irreducible if the monic GCD $\Delta_{H}(z)$ of all its $n \times n$ minors is one, or equivalently, if $\operatorname{rank} H(\lambda)=n$, for all $\lambda \in C$,

2) $H(z)$ is said to be column-reduced if its highest column-degree coefficient matrix has full rank [15, p. 384],

3) $H(z)$ is said to be unimodular under the condition $m=n$, if its determinant is a nonzero constant.

We shall establish a fundamental structure of an equalizable channel system as follows:

Theorem 2 (Irreducible-Paraunitary Factorization): Let $H(z) \in C[z]^{m \times n}$ be equalizable with $m \geq n$, and let $\Delta_{H}(z)=z^{\ell}$ where $\Delta_{H}(z)$ is the monic GCD of all the $n \times n$ minors of $H(z)$. Then it can be factorized as

$$
H(z)=H_{I}(z) H_{P}(z)
$$

where $H_{I}(z) \in C[z]^{m \times n}$ is irreducible with $\operatorname{deg}$ $H_{I}(z) \leq \operatorname{deg} H(z)$, and $H_{p}(z) \in C[z]^{n \times n}$ is paraunitary with $\operatorname{deg} H_{p}(z)=\ell$. Moreover, the above factorization (15) is unique up to the multiplication of a unitary matrix $U \in C^{n \times n}$.

Henceforth, in the above factorization (15), $H_{I}(z)$ is said to be an irreducible factor of $H(z)$, and $H_{p}(z)$ is said to be a paraunitary factor of $H(z)$.

Some comments on Theorem 2 are given below.

1) The paraunitary transfer function $H_{p}(z)$ has a factorization given as

$$
H_{p}(z)=U_{0} \Phi(z) U_{1} \Phi(z) \cdots U_{\ell-1} \Phi(z) U_{\ell},
$$


where $U_{k} \in C^{n \times n}, k=\overline{0, \ell}$ are unitary matrices, and

$$
\Phi(z):=\left[\begin{array}{cc}
I_{n-1} & 0 \\
0 & z
\end{array}\right] \in C[z]^{n \times n} .
$$

2) By the uniqueness of the factorization of (15), we mean that if $H(z)$ has another factorization

$$
H(z)=\tilde{H}_{I}(z) \tilde{H}_{p}(z),
$$

where $\tilde{H}_{I}(z) \in C[z]^{m \times n}$ is an irreducible FIR system, and $\tilde{H}_{p}(z) \in C[z]^{n \times n}$ is a paraunitary FIR system, then there exists a unitary matrix $U \in C^{n \times n}$ such that

$$
\tilde{H}_{I}(z)=H_{I}(z) U \text { and } \tilde{H}_{p}(z)=U^{H} H_{p}(z) .
$$

The fact that $U$ is constant is non-trivial.

3) It is well known that any infinite impulse response (IIR) transfer matrix $H(z)$ with $m \geq n$ has a minimum phase-allpass factorization as follows: $H(z)=H_{m}(z) H_{a}(z)$, where $H_{m}(z)$ is minimum phase and $H_{a}(z)$ is allpass, and both are IIR filters [14]. However, such a factorization in general does not exist for the FIR case. Theorem 2 states that any FIR $H(z)$ has a factorization of an FIR irreducible factor and an FIR paraunitary factor if $H(z)$ is equalizable.

4) Recent proposed subspaced-based methods [4]-[7] and linear predication methods [8] consider the special case when the channel system is irreducible. The irreducibility condition on $H(z)$ implies that $H(0)$ has full column rank. This condition imposes a restrictive condition on the arrival time from the sources to the receivers. For example, if one signal arrived later than the others, then its corresponding column of $H(0)$ would be zero, and hence $H(0)$ could not be of full column rank.

5) In a different context, Vaidyanathan [13] has considered the case when $H(z)$ is square. This class may be general enough for his purposes, but it is a very restrictive for channel systems that is equalizable. Theorem 2 extends mathematically from the square matrix case to the tall matrix case. However, in applications, it extends from the very restrictive class to the most general class of equalizable channel systems. Also, the unimodular factor in his factorization has been extended to the irreducible factor in our presentation. In addition, we have shown the uniqueness of this factorization, which is important for future developments.

Theorem 2 provides a system theoretical foundation for the investigation of the problem of blind channel equalization in the most general form. It includes, for example, Rayleigh-fading channels in a mobile communication system in which the signals arrive randomly with delays together with channel orders varying with times.

Based on Theorem 2, a necessary and sufficient condition for the existence of strict deconvolvers for $H(z)$ will be presented below, which shows one of limits of SOS-based approaches to the solution of the blind equalization problem.

Theorem 3: Let $H(z)$ be equalizable and $\{s(t)\}$ be zero-mean and white, and let $H(z)$ has the irreducible-paraunitary factorization as follows: $H(z)=H_{I}(z) H_{p}(z) \in C[z]^{m \times n}$. If $W_{I}(z) \in C[z]^{m \times n}$ is any irreducible whitener for $H(z)$, then it is a strict deconvolver for $H_{I}(z)$. Moreover, any strict deconvolver for $H_{I}(z)$ is also deconvolver for $H(z)$, if and only if, the paraunitary factor $H_{p}(z)$ of $H(z)$ is represented as $H_{p}(z)=U \Lambda(z)$, where $U \in C^{n \times n}$ is unitary and $\Lambda(z) \in \Lambda[z]^{n \times n}$.

It follows immediately from Theorem 3 an important limitation of second order statistics when the input sequence is white.

Corollary : Let $H(z)$ be equalizable and $\{s(t)\}$ be zero-mean and white. A channel system $H(z)$ can be deconvolved by an irreducible whitener if and only if it has the form $H(z)=H_{I}(z) U \Lambda(z)$.

Some comments on Theorem 3 are given below.

1) When $H(z)$ is connected with an irreducible whitener $W_{I}(z)$ in a cascade, the resulting system will have the form

$$
W_{I}(z) H(z)=W_{I}(z) H_{I}(z) H_{P}(z)=D \tilde{H}_{P}(z),
$$

where $D$ is a regular diagonal constant matrix and $\tilde{H}_{P}(z)$ is paraunitary. As such, we need only to 
consider the equalization problem of a paraunitary system.

2) When the input sequence is white, the best for second-order statistics can do is to deconvolve the channel. One must use higher-order statistics for the equalization.

\section{CONCLUSIONS}

We considered a class of FIR MIMO channel systems called equalizable channels with transmission zero at the origin $z=0$. We formulated the blind equalization problem of an FIR MIMO channel system, and showed a necessary and sufficient condition for the existence of an FIR equalizer. To analyze the FIR channel system, we showed that any equalizable FIR system could be written as a product of an irreducible FIR system and a paraunitary FIR system. Based on this fact, we showed that any equalizable FIR channel system could be reduced to a paraunitary FIR system by any irreducible whitener of channel system.

Noise $n(t)$ is ignored in this paper for the purpose of analysis. A remaining problem is to estimate a degree of such noise corruption in blind channel equalization. Another remaining problem is to consider how to utilize the notion of blockers for equalizing a channel system (which is not irreducible nor columnreduced) driven by colored input sources.

\section{REFERENCES}

[1] Special Issue on Signal Processing for Advanced Communications, IEEE Trans. Signal Processing, vol. 45, Jan. 1997.

[2] L. Tong, G. Xu, and T. Kailath, "A new approach to blind identification and equalization of multipath channels," in Proc. 25th Asilomar Conf., Pacific Grove, CA, 1991, pp. 856-860.

[3] L. Tong, G. Xu, and T. Kailath, "Fast blind equalization via antenna arrays." In Proc. ICASSP, 1993, vol. 4, pp. 272-275.

[4] E. Moulines, P. Duhamel, J. Cardoso, and S. Mayrargue," Subspace methods for blind identification of multichannel FIR filters," IEEE Trans. Signal Processing, vol. 43, pp. 516-525, Feb. 1995.

[5] K. Abed Merain, P. Loubaton, and E. Moulines, "A subspace algorithm for certain blind identification problem," IEEE Trans. Inform. Theory, vol. 43, pp. 499-511, March 1997.
[6] K. Abed Merain, J. Cardoso, A. Gorokhov, P. Loubaton, and E. Moulines, "On subspace methods for blind identification of single-input multiple-output FIR systems," IEEE Trans. Signal Processing, vol. 45, pp. 42-55, Jan. 1997.

[7] A. Gorokhov and P. Loubaton, "Subspace-based techniques for blind separation of convolutive mixtures with temporally correlated source," IEEE Trans. Circuits Syst.-I, vol. 44, pp. 813820, Sept. 1997.

[8] D. T. Slock, "Blind fractionally-spaced equalization, perfect reconstruction filter-banks and multichannel linear prediction," in Proc. ICASSP, vol. 4, pp. 585-588, 1994.

[9] Y. Inouye and R. Liu, "Criterion for direct blind deconvolution of MIMO FIR systems driven by white source signals," in Proc. ICASSP, Phoenix, AZ, 1999, vol. 5, pp. 2889-2892.

[10] R. Iiu and Y. Inouye, "Direct signal separation of convolutive mixtures of white spatially independent nongaussian signals," in Proc. ICA'99 (1st Int. Workshop on Independent Component Analysis and Signal Separation), Aussois, France, pp. 233-238, 1999.

[11] R. Liu and Y. Inouye, "Direct blind deconvolution of multiuser-multichannel systems," in Proc. ISCAS, Orlando, FL, vol. 5, pp. 49-52.

[12] J. K. Massey and M. K. Sain, "Inverse of linear sequential circuits," IEEE Trans. Computers, pp. 330-337, 1968.

[13] P. P. Vaidyanathan, "How to capture all FIR perfect reconstruction QMF banks with unimodular matrices ?," in Proc. ICASSP, New Orleans, LA, May 1990, pp. 2030-2033.

[14] Y. Inouye, "Notes on minimum-energy delay property of impulse-response sequence of minimum-phase transfer functions," IEEE Trans. Circuits Syst, vol. CAS-34, pp. 188-190, Feb. 1987.

[15] T. Kailath, Linear Systems. New York: Prentice-Hall, 1980.

[16] G. D. Forney, "Minimal bases of rational vector spaces with, applications to multivariable linear systems," SIAM J. Control, vol. 13, no. 3, pp. 493-520, May 1975. 